\title{
CLIMA PSICOLÓGICO COMO PREDITOR DA SAÚDE E DO BEM-ESTAR DE PROFISSIONAIS DE SAÚDE EM CONTEXTO HOSPITALAR
}

\author{
Isabel Silva Carvalho ${ }^{1}$
}

Resumo: O clima psicológico, enquanto representação psicológica do ambiente de trabalho, ou seja, das estruturas, processos e acontecimentos proximais (Rousseau, 1988) e reflexo dos processos de atribuição de sentido às experiências de trabalho (Weick, 1995), tem sido considerado como uma variável explicativa importante do comportamento, saúde e bem-estar dos indivíduos nas organizações (Parker, Baltes, Young, Huff, Altmann, Lacost \& Roberts, 2003). Este estudo, com uma amostra de 375 profissionais de saúde hospitalar, tem como objectivo a análise da relação entre clima psicológico e a saúde e o bem-estar. Tem como nível de análise o indivíduo (Reichers \& Schneider, 1990), e como referencial teórico o modelo de Jones \& James (1979). A saúde e o bem-estar são avaliados através da percepção da saúde física e psicológica, vitalidade e funcionamento cognitivo e social. Os resultados apontam para o papel do clima psicológico enquanto preditor da saúde e bem-estar, bem como para o papel de mediação da satisfação com o trabalho nesta relação. São discutidas as implicações nas intervenções de saúde ocupacional cujo objectivo seja a melhoria da qualidade de vida no trabalho.

Palavras-chave: clima psicológico, saúde, bem-estar, satisfação com o trabalho, profissionais de saúde.

Psychological climate as a predictor of hospital professionals' health and well-being (Abstract): Psychological climate as a proximal representation of organizational structures, processes and events (Rousseau, 1988) as well as a meaning attribution processes in the work context (Weick, 1995) as been reported as an important predictor of individual beahviour, health, and well-being in organizations (Parker, Baltes, Young, Huff, Altmann, Lacost \& Roberts, 2003). In this study we examine the relationship between psychological climate and health and well-being of health care professionals. Drawing on Jones and James (1979) model of psychological climate, several facets of climate are measured as well as multiple dimensions of health and well-being: perception of physical health, vitality, cognitive functioning,

\footnotetext{
${ }^{1}$ Instituto Superior de Ciências do Trabalho e da Empresa, Lisboa. Departamento de Psicologia Social e das Organizações. isabel.carvalho@iscte.pt.
} 
social functioning, and affective well-being. Results suggested that psychological climate is a strong predictor of employees health and well-being. Results also suggested that work satisfaction mediates the relationship between perceptions of work and environment characteristics and health and well-being. Practical implications are discussed and interventions to improve quality of work life are suggested.

Key-words: psychological climate, health, well-being, work satisfaction, health care professionals.

\section{“(...) an ounce of prevention is worth a pound of} cure.” Levi, 1982

\section{Introdução}

A saúde e o bem-estar relacionados com o trabalho são uma dimensão da vida humana que progressivamente tem vindo a interessar investigadores do comportamento humano, da gestão e da saúde pública, entre outros.

A principal razão prende-se com a presença do trabalho na vida das pessoas e com os factores de risco que ele comporta. Em 1995, 45\% da população mundial estava enquadrada profissionalmente (World Health Organization [WHO], 1996) e estima-se que, na União Europeia, esse valor atinja os 70\% em 2010, de acordo com os objectivos estabelecidos para o desenvolvimento económico na Agenda de Lisboa (European Community, [EU], 2005).

Paralelamente, uma proporção considerável e crescente da população trabalhadora tem a sua saúde e bem-estar em risco, em consequência directa ou indirecta do seu trabalho (WHO, 2004), e só na União Europeia esta proporção representa um terço da força de trabalho (Ivanov, 2005).

Esta realidade tem custos que se fazem sentir transversalmente, em todos os quadrantes da sociedade, não só porque implicam com a qualidade de vida dos indivíduos (Jamison, Wallace, \& Jamison, 2004) e com o desempenho e a eficiência organizacional (Almond \& Healey, 2003), mas porque impõem custos adicionais na economia que podem, inclusive, pôr em causa a sustentabilidade dos sistemas de protecção social (McDaid, Curran \& Knapp, 2005).

Em Portugal, os dados do relatório sobre a Saúde no Trabalho, na União Europeia, apontam como segundo maior problema relacionado com o trabalho o stress, a depressão e a ansiedade, logo a seguir às perturbações 
músculo-esqueléticas (EU, 2004). Segundo o estudo de Ramos e Sennfelt (1996) sobre os custos da depressão em Portugal, 80\% do custo económico deveu-se à perda de produtividade.

O sector de actividade com maior prevalência de casos é o da Saúde e da Educação, destacando-se o da Saúde pelo maior número de casos com duas semanas ou mais de absentismo-doença (EU, 2004).

Muita da investigação que tem sido realizada sobre a relação do trabalho com a saúde e o bem-estar tem sido desenvolvida com base no conceito de stress no trabalho, que, de forma abrangente, se refere às experiências psicológicas de desconforto e dificuldade, envolvendo estados emocionais negativos, como a ansiedade, frustração, raiva e desconforto, entre outros (Lazarus, 1999) que resultam do desequilíbrio ou discrepância entre níveis percebidos ou desejados de um determinado aspecto do ambiente de trabalho e os níveis percepcionados (Warr, 1990).

Têm-se baseado em indicadores como a sintomatologia física, a prevalência de perturbação mental e doença (Shirom, 2003), sintomas adicionais como as perturbações do sono, problemas cardiocirculatórios ou gastrointestinais (Aasland, Olff, Schweder \& Ursin, 1997) ou ainda em estilos de vida e comportamentos de risco, como consumo tabaco, álcool e drogas (Grunberg, Moore \& Greenberg, 1998). Esta hipótese de que o trabalho é um factor causal de perturbação da saúde física e psicológica tem sido suportada por vários estudos longitudinais (Siegrist, 1996; Sonnentag \& Frese, 2004; Theorell \& Karasek, 1996).

Esta investigação têm-se centrado predominantemente nos efeitos negativos "distress" (negligenciando os efeitos positivos "eustress") que o trabalho exerce sobre as pessoas (Diener, Suh, Lucas \& Smith, 1999) e com os factores organizacionais que estão na sua origem (Danna \& Griffin, 1999; Ganster \& Schaubroeck, 1991). Assenta no modelo clássico médico e na necessidade de reparar/tratar o disfuncionamento e a patologia, negligenciando a prevenção dos riscos e a promoção dos factores protectores (Griffiths \& Schabracq, 2003).

Esta abordagem, que enfatiza a disfunção, tem proporcionado muita evidência científica sobre as causas e consequências de ambientes de trabalho deletérios para a saúde e bem-estar dos trabalhadores. Todavia, pouco tem contribuído para explicar o outro lado da realidade, nomeadamente alguns dados obtidos no Third European Survey on work Conditions 2000 (EU, 2004), como, por exemplo, porque é que oitenta e quatro por cento dos trabalhadores europeus estão satisfeitos com as suas condições de trabalho e sessenta por cento não tiveram nenhum episódio de absentismo-doença no ano anterior. Com o conhecimento acumulado, é possível explicar como é que o sofrimento psicológico pode estar associado ao absentismo (Almond \& Healey, 2003), mas não como é que o bem-estar está associado com a 
retenção de trabalhadores e com o bom desempenho profissional, como sugere o estudo de Wright e Bonett (1992).

Tendo presente o movimento da psicologia positiva, que apela para o estudo do potencial dos fenómenos positivos (Seligman \& Csikszentmihalyi, 2000), e as dinâmicas subjacentes ao desempenho excepcional, quer das pessoas, quer das organizações (Cameron \& Caza, 2004), e ainda a recomendação de Griffith e Schabracq (2003) de que a psicologia da saúde no trabalho deve focar-se mais na saúde e bem-estar positivo, este estudo tem como objectivo analisar a relação entre as percepções sobre o ambiente de trabalho e a saúde, bem como explorar a forma como este processo se desenrola, analisando o papel mediador da satisfação com o trabalho (Ostroff, Kinicki \& Tamkis, 2003).

Para atingir estes objectivos, optou-se por abordar tanto o clima psicológico como a saúde enquanto conceitos multidimensionais, usando uma perspectiva de banda larga dos fenómenos em estudo que dê conta simultaneamente da quantidade e complexidade da informação que se pretende obter num dado momento (Cronbach \& Gleser, 1965).

Neste sentido, vai ser usada uma concepção da saúde enquanto estado de completo bem-estar físico, mental e social (WHO, 1948), englobando aspectos como a vitalidade ou energia e a eficácia no desempenho (Larson, 1999). Este conceito vai para além da ausência de doença, salientando a présença de algo positivo (Keyes, 2005; Ryff \& Singer, 1998).

O ambiente de trabalho vai ser abordado tendo como referência o conceito de clima psicológico enquanto conceito multidimensional que engloba as representações psicológicas significativas dos acontecimentos, prócessos e estruturas organizacionais próximas do indivíduo (James, Hater, Gent \& Bruni, 1979; Rousseau, 1988). Estas percepções representam a forma como o indivíduo se apropria e representa cognitivamente o ambiente de trabalho em termos do seu significado e importância (James \& Jones, 1974; James \& Sells, 1981). Pressupõe que os indivíduos respondem aos estímulos do ambiente de acordo com a forma como os percebem e a componente mais importante é o sentido ou sentidos que estes lhes atribuem (James, James, \& Ashe, 1990). Neste sentido, as percepções do ambiente de trabalho serão avaliadas, tendo como nível de teoria, medida e análise o indivíduo (Reichers \& Schneiders, 1990; Rousseau, 1988), o que nos situa claramente no conceito de clima psicológico e não no de clima grupal ou colectivo (por Serviços) ou clima organizacional (o hospital como um todo).

Para perceber como é que as pessoas chegam a conclusões gerais sobre o ambiente de trabalho, usámos como referência o modelo de Jones e James (1979), que, após reverem a literatura sobre as relações conceptuais entre estrutura organizacional e atitudes e comportamentos dos trabalhadores, propuseram uma categorização do clima psicológico. Esta tem em conta 
a forma como os indivíduos representam cognitivamente o seu ambiente de trabalho e, por isso, usam como referente as características situacionais do trabalho: características do trabalho (ambiguidade, conflito e sobrecarga); características do papel (ênfase nos objectivos, suporte, influência na decisão; grupo de trabalho e ambiente social (cooperação, orgulho) e atributos organizacionais e de subsistema (inovação e abertura à informação) (James \& Jones, 1974, 1976).

A escolha do clima psicológico enquanto referência conceptual visa também ultrapassar uma tendência da investigação actual que enfatiza as diferenças individuais, salientando as avaliações, bem como os estilos de coping idiossincráticos de cada indivíduo. Esta abordagem tem negligenciado o facto de que o ambiente social é uma realidade poderosa, socialmente construída (Ashforth, 1985; Ashkanasy, Wilderon \& Peterson, 2000; Schneider \& Reichers, 1983), onde as pessoas partilham regras de avaliação (Averill, 1986) e formas de lidar com o mundo (Semmer, 2003), com base em significados partilhados ou representações sociais (Moscovici, 1984).

Esta perspectiva subjectiva, que nos leva a considerar o clima como um conceito molar (por oposição a um referente específico, como, por exemplo o clima de inovação ou o clima de segurança), reflecte o processo de atribuição de sentido (Weick, 1995) pelo qual os membros de uma organização entendem e partilham colectivamente as suas experiências organizacionais. Estas interpretações são propriedade de um colectivo social e são concomitantes com os processos de interacção social entre os membros organizacionais (Ashforth, 1985; Rentsch, 1990; Schneider \& Reichers, 1983).

Esta abordagem permite-nos considerar simultaneamente um conjunto alargado de características do trabalho (Van Veldhoven, Taris \& Jonge, 2005) que consistentemente têm sido identificadas como importantes preditoras da saúde e bem-estar relacionados com o trabalho (De Lange, Taris, Kompier, Houtman \& Bongers, 2004; Lee \& Ashforth, 1996), nomeadamente as exigências do trabalho, sejam físicas, emocionais ou cognitivas (Jonge, Dormann, Janssen, Dollard, Landeweerd \& Nijhuis, 2001), o controlo (latitude de decisão) sobre elas (Karasek, 1979), a autonomia (Hackman \& Oldham, 1980), o suporte social (Viswervaran, Sanchex \& Fisher, 1999), o feedback sobre o desempenho e o conflito de papel (Parker, Wall \& Cordery, 2001). Por exemplo, a análise dos dados do estudo Whitehall II (Stansfeld, Head \& Marmot, 2000) revela que as exigências elevadas do trabalho são um bom preditor da diminuição da saúde, bem como da doença mental e que a falta de controlo no trabalho está moderadamente associada ao risco de dependência alcoólica. Por outro lado, o suporte social e o controlo no trabalho demonstraram exercer um efeito protector na saúde mental e no funcionamento saudável, bem como uma associação com menos episódios de absentismo-doença. 
Neste sentido, a nossa primeira hipótese é que a percepção sobre o ambiente de trabalho influencia a percepção da saúde e, quanto mais positiva for, melhor será a avaliação da saúde.

Na relação entre as percepções do ambiente de trabalho e os comportamentos e atitudes do trabalhador, uma das lacunas que têm sido identificadas é a do papel das variáveis intervenientes neste processo (Kopelman, Brief \& Guzzo, 1990; Ostroff, Kinicki \& Tamkins, 2003).

Estudos recentes de meta-análise têm identificado alguns estados afectivos e cognitivos como mediadores na relação entre clima psicológico e desempenho, nomeadamente, a motivação e a satisfação com o trabalho, (Carr, Schmidt, Ford \& DeShon, 2003; Parker, Baltes, Young, Huff, Altman, Lacost \& Roberts, 2003). Esta mediação encontra suporte na teoria do comportamento planeado, de Ajzen e Fishbein (1980), que sugere que os estados afectivos e cognitivos que resultam das percepções do ambiente de trabalho, combinados com as oportunidades para agir e associando-se às crenças, se podem transformar nos antecedentes imediatos do comportamento (Mathieu \& Zajac, 1990). Apesar de ainda não ter sido estabelecido com precisão o mecanismo pelo qual este processo ocorre, tem sido sugerido que o ambiente organizacional actue como influência normativa, ao modelar o sistema de crenças (Wiener, 1982).

Nos estudos de meta-análise referidos, não foi possível analisar a influência destas variáveis mediadoras nos estudos que relacionam clima psicológico e saúde, porque os estudos que as consideram são muito escassos.

Tentaremos contribuir para colmatar esta lacuna, analisando se, por um lado, existe evidência que relacione percepções sobre o ambiente de trabalho com a satisfação e, por outro, se a satisfação se relaciona com a saúde e, se for esse o caso, analisaremos o papel mediador da satisfação na relação entre clima psicológico e saúde.

A satisfação com o trabalho tem sido estudada enquanto "estado emocional positivo ou agradável que resulta da avaliação do trabalho ou da experiência de trabalho" (Locke, 1976, p. 130) ou ainda como a resposta afectiva à situação de trabalho (Brooke, Russell \& Price, 1988).

Esta avaliação tem sido considerada com podendo ser uma síntese, onde o indivíduo reconcilia diferentes facetas de forma sumária, e a que, portanto, se pode aceder através de um único item (Wanous, Reichers \& Hudy, 1997), ou como sendo uma avaliação parcelar de distintos aspectos só possível de ser acedida através de medidas compósitas (Mullarky, Wall, Warr, Clegg, \& Stride, 1999), por exemplo, a especificidade referida por Warr (1999), entre aspectos extrínsecos (aspectos instrumentais ou materiais, como salário, a segurança do emprego ou as possibilidades de promoção) e aspectos intrínsecos (qualidade do trabalho como as relações com colegas e supervisor ou o grau de autonomia). 
As características do trabalho parecem ser um bom preditor da satisfação com o trabalho (Day \& Bedeian, 1991; Gunter \& Furnham, 1996; Repetti \& Cosmas, 1991). Por exemplo, as exigências do trabalho estão negativamente associadas com a satisfação, num estudo longitudinal com profissionais de saúde (Jonge et al., 2001), e as relações de apoio no trabalho estão positivamente associadas com a satisfação, nestes profissionais (Jonge, Janssen \& Breukelen, 1996).

Para analisar a causalidade, James e Tetrick (1996) compararam três modelos explicativos e os resultados que obtiveram apontam claramente para o papel explicativo das características do trabalho na satisfação, sendo pouco evidente a relação inversa.

Com base nesta evidência, a segunda hipótese a ser testada pressupõe que, quanto mais positivas forem as percepções sobre o ambiente de trabalho, mais positiva será a satisfação.

Paralelamente, o stress relacionado com o trabalho conceptualizado enquanto conjunto de efeitos negativos resultantes da relação com o trabalho, tem sido apontado com uma das causas de satisfação reduzida (Cass, Siu, Faragher \& Cooper, 2003; Grebner, Semmer \& Elfering, 2005). Por exemplo, LaRocco, House e French (1980) referem que ambientes de trabalho muito exigentes e satisfação podem ser os melhores factores explicativos da temsão psicológica. Entre pessoal médico, Tetrick e LaRocco (1987) obtiveram evidência no sentido da satisfação estar negativamente associada à depressão e ansiedade relacionadas com o trabalho. Sutton e Kahn (1986) referem que a insatisfação não só medeia a relação entre percepções do ambiente de trabalho e níveis elevados de tensão, como aumenta directamente essa tensão psicológica. Por outro lado, uma revisão recente da literatura refere que "os estados emocionais positivos podem promover percepções saudáveis, crenças e inclusive o próprio bem-estar físico" (Salovey et al., 2000).

Seguindo a proposta de Kopelman, Brief e Guzzo (1990), que desenvolve o modelo de Jones e James (1979) e propõe que o impacto das percepções do clima nos comportamentos e atitudes individuais e organizacionais (desempenho e produtividade) pode ser mediada por estados afectivos e cognitivos (satisfação e motivação), a nossa terceira hipótese é a de que a relação do clima com a saúde seja mediada pela satisfação.

\section{Método}

\section{Amostra e Procedimento}

Nesta investigação participaram 366 profissionais de saúde, que correspondem a $18,2 \%$ da população, de um hospital central da região de Lisboa. A idade dos participantes varia entre 19 e 65 anos, sendo a média de 38 
anos. Destes, 71 por cento são do sexo feminino. No que diz respeito à categoria profissinal, os enfermeiros correspondem a 35 por cento da amostra, os médicos constituem 15 por cento, os técnicos superiores de saúde correspondem a 12 por cento, os administrativos a 16 por cento e o pessoal de apoio corresponde a 22 por cento da amostra.

Os dados foram recolhidos por meio de um questionário de autopreenchimento entregue em mão no local de trabalho, juntamente com uma carta explicativa do estudo e das garantias de confidencialidade e com um envelope selado dirigido à Faculdade de Psicologia e de Ciências da Educação da Universidade de Lisboa. Previamente à recolha de informação, foi feita uma campanha de informação e sensibilização para a importância da saúde ocupacional e deste estudo em particular, que incluiu o envio de uma carta a todos os profissionais, junto com o recibo de vencimento, a afixação de cartazes em todos os serviços do hospital e a realização de sessões de esclarecimento durante as reuniões de equipa em cada serviço, tudo com o fim de explicar os objectivos e metodologia do estudo.

\section{Instrumentos}

\section{Clima Psicológico}

As percepções sobre o clima psicológico foram obtidas tendo em conta 15 variáveis que se organizam em cinco dimensões, seguindo o modelo proposto por Jones e James (1979), e que, de seguida, se descrevem.

Foi realizada uma Análise Factorial em Componentes Principais (ACP) com rotação varimax e um limite máximo de 5 factores. $\mathrm{O}$ modelo obtido a partir da correlação dos items e cada um dos 5 factores correspondeu, genericamente, ao modelo proposto por Jones e James (1979) e explicava cerca de $51 \%$ da variância total.

Em seguida foi efectuada uma ACP em cada uma das dimensões, por forma a identificar as facetas que as compõem. Obtivemos soluções sem itens com baixa comunalidade, nem itens mistos (com uma saturação igual ou superior a $0,45 \mathrm{em}$ dois ou mais factores). Obtiveram-se cinco dimensões: atributos organizacionais e de subsistema, grupo de trabalho e ambiente social, características da liderança, características do papel e caracteristicas do trabalho, que se desecrevem de seguida.

A dimensão atributos organizacionais e de subsistema inclui 7 facetas: comunicação, avaliação do desempenho, resposta à mudança, conflito e cooperação, formação, liberdade e distribuição de recursos.

A Comunicação, medida através de uma escala com 5 itens, refere-se à qualidade da comunicação interna ("A comunicação neste hospital é muito boa", "As diferentes equipas não partilham, entre si, informação sobre o que se está a passar"); 
a Avaliação do Desempenho, medida com 5 itens, e refere-se ao modo e frequência com que o trabalho dos profissionais é avaliado ("De um modo geral, é difícil para qualquer profissional avaliar a qualidade do seu desempenho neste hospital", "A forma como as pessoas realizam o seu trabalho raramente é avaliado");

a Resposta à Mudança, medida com 6 itens, reflecte a capacidade para mudar procedimentos em resposta a novas exigências e resolver problemas, à medida que eles surgem ("O hospital responde rapidamente às necessidades de mudança", "O hospital é rápido a descobrir que as coisas têm de ser feitas de uma maneira diferente");

o Conflito e Cooperação, medido com 6 itens, descreve o conflito e as práticas de cooperação ("Estão sempre a acontecer conflitos no hospital", "As pessoas neste hospital trabalham todas no mesmo sentido");

a Formação, medida com 6 itens, refere-se à formação recebida pelos profissionais no hospital necessária à realização do seu trabalho ("As pessoas não recebem formação adequada quando são introduzidos novos procedimentos", "As competências dos profissionais são desenvolvidas para que estes possam melhorar o seu desempenho");

a Liberdade, medida com 6 itens, refere-se à liberdade que os profissionais têm para fazer o seu trabalho sem interferência de superiores ("Os supervisores fazem um controlo apertado do trabalho", "Os superiores permitem que os profissionais façam o seu trabalho da maneira que acharem melhor");

e a Distribuição de Recursos, medida com 7 itens, que diz respeito à presença dos recursos físicos necessários para a realização do trabalho ("Os profissionais neste hospital estão bem apoiados em termos de equipamentos", "No hospital há, muitas vezes, falta de material").

As respostas eram dadas numa escala de 5 pontos, que variava entre 1 ("Discordo totalmente") e 5 ("Concordo totalmente"). Estas escalas foram desenvolvidas por Lawthorn, Patterson e West (1996),

A dimensão grupo de trabalho e ambiente social inclui uma única faceta denominada Apoio dos Colegas, medida com 4 itens a adaptados de West e Savage (1988), e diz respeito à medida em que os profissionais recebem apoio ou ajuda dos colegas na realização do seu trabalho ("Posso contar com os colegas para me ouvirem quando preciso de falar sobre problemas de trabalho", "Posso contar com os colegas para me ajudarem numa situação de crise no trabalho, ainda que isso implique desviarem-se do seu trabalho normal"). As respostas eram dadas numa escala de 5 pontos, que variava entre 1 ("Raramente") e 5 ("Totalmente").

A dimensão características da liderança inclui o apoio do supervisor, medido com 6 itens, adaptados de West e Savage (1988) e refere-se ao apoio que cada profissional recebe do seu superior hierárquico directo 
("Posso contar com o meu superior hierárquico quando preciso de falar sobre problemas do trabalho", "Posso contar com o meu superior hierárquico para me ajudar a resolver uma tarefa difícil"); e a Liderança Participativa, adaptada de Chambel e Curral (1998), medida com 4 itens, que diz respeito à influência exercida pelos profissionais, sobre as decisões relativas à organização do trabalho ("O seu superior directo pede a sua opinião antes de tomar uma decisão que afecta o seu trabalho", "Posso participar nas decisões que me vão afectar"). As respostas eram dadas numa escala de 5 pontos, que variava entre 1 ("Nunca") e 5 ("Quase sempre").

A dimensão características do papel inclui a Clareza de Papel, medida por 5 itens, adaptados do questionário desenvolvido por Breaugh e Colihan (1994), os quais expressam o grau em que os participantes consideram que aquilo que deles é esperado no trabalho está claro ("Os objectivos do meu trabalho estão claramente definidos", "Eu sei quais são as minhas responsabilidades"); o Conflito de Papel, medido por uma escala com quatro itens, adaptada do questionário desenvolvido por Rizzo, House, e Lirtzman (1970), expresso pela existência de pedidos incompatíveis aos profissionais ("Eu recebo ordens de duas ou mais pessoas", "Outros profissionais pedem-me para fazer coisas incompatíveis entre si"); e a Sobrecarga de Papel, uma escala com cinco itens, adaptada de Caplan (1994), que reflecte a medida em que a quantidade de trabalho é percepcionada como excessiva ("Eu não tenho tempo suficiente para fazer o meu trabalho", "Não consigo dar resposta a todos os pedidos que me fazem durante o trabalho"). As respostas eram dadas numa escala de 5 pontos, que variava entre 1 ("Nunca") e 5 ("Quase sempre").

A dimensão características do trabalho inclui o Feedback sobre o Desempenho, adaptado do Job Diagnostic Survey de Hackman e Oldham, 1975, medido com 4 itens, que diz respeito à informação que os profissionais recebem sobre a qualidade do seu trabalho ("Geralmente sei se o meu trabalho está ou não bem feito", "Frequentemente tenho dificuldades em perceber se estou a fazer um bom trabalho"); uma faceta denominada Latitude de Decisão, adaptada do Job Diagnostic Survey de Hackman e Oldham (1975), medida com 5 itens, que diz respeito à medida em que os profissionais planeavam e escolhiam a forma de realizar o seu trabalho ("Em que medida determina os métodos e procedimentos que usa no seu trabalho?", "Em que medida planeia o seu trabalho?). As respostas eram dadas numa escala de 5 pontos, que variava entre 1 ("Raramente") e 5 ("Totalmente").

Satisfação com o Trabalho

A satisfação com o trabalho foi medida com uma escala de 16 itens desenvolvida por Mullarkey, Wall, Warr, Clegg e Stride (1999). Foi pedido aos participantes para avaliarem a sua satisfação com vários aspectos do seu 
trabalho, como, por exemplo, as condições físicas de trabalho, a liberdade para escolher métodos de trabalho e a formação, numa escala de 7 pontos, que variava entre 1 ("Extremamente insatisfeito") e 7 ("Extremamente satisfeito").

A Saúde

A saúde, enquanto variável multidimensional, foi avaliada com escalas referentes a várias dimensões da saúde. Uma escala com 4 itens adaptada do Health Status Questionnaire (SF-36), (McHorney, Ware \& Raczeck, 1993) avaliava a percepção sobre o estado de saúde em geral. Os participantes deviam relatar em que medida se consideravam saudáveis ("Eu sou tão saudável como qualquer outra pessoa", "De um modo geral diria que a minha saúde é fraca"), numa escala que variava entre entre 1 ("Totalmente falso") e 5 ("Totalmente verdadeiro").

Uma escala com 6 itens, adaptada de Warr (1990), avaliava o bem-estar psicológico. Os participantes deviam avaliar a frequência com que, no último mês, se tinham sentido "tensos" e "abatidos", numa escala de 6 pontos, que variava entre 1 ("Nunca") e 6 ("Todo o tempo"). Uma escala com 8 itens avaliava o funcionamento social. Esta escala é uma adaptação do Health Status Questionnaire (SF-36, McHorney, Ware \& Raczeck, 1993) e do Modified Self-Report social Adjustment Scale (Cooper, Osborn, Gath \& Feggetter, 1982). Os participantes deviam avaliar a medida em que a sua saúde os impediu de realizar o seu trabalho ou outras actividades sociais ("Fez menos do que gostaria", "Esteve limitado no tipo de trabalho ou em outras actividades sociais"), numa escala de 5 pontos, que variava entre 1 ("De maneira nenhuma") e 5 ("Completamente"). Uma escala com 6 itens avaliava a vitalidade. Os participantes deviam avaliar em que medida se tinham sentido com energia ou, pelo contrário, cansados, numa escala de 6 pontos, que variava entre 1 ("Sempre") e 6 ("Nunca"). Uma escala de 4 itens, retirada do General Health Questionnaire (Goldberg \& Williams, 1991), avaliava o funcionamento cognitivo ("Tem sido capaz de se concentrar no que está a fazer", "Tem sido capaz de tomar decisões sobre as coisas"). Os participantes deviam avaliar a medida em que se sentiam capazes de tomar decisões e resolver problemas, numa escala de 4 pontos, que variava entre 1 ("Pior do que o costume") e 4 ("Melhor do que o costume").

\section{Resultados}

Na Tabela 1, estão descritas as médias, desvio-padrão e correlações entre as variáveis e na diagonal os Alphas de Cronbach $(\alpha)$ das escalas. A maioria das escalas revela boa consistência interna, com $\alpha$ bons acima de 
0,70, ou satisfatórios, acima de 0,60 . A maioria das variáveis estão significativamente correlacionadas entre si. Adoptámos as convenções sugeridas por Cohen e Cohen (1983), para considerarmos as correlações significativas até 0,20 como fracas, entre 0,21 e 0,40 como moderadas e acima de 0,40 como elevadas. Todas as facetas do clima apresentam correlações significativas com a satisfação e com a saúde. Em relação à satisfação, destacam-se as correlações elevadas e positivas com o apoio do supervisor $(r=0,49$, $p<0,01)$, a liderança participativa $(r=0,37, p<0,01)$, e o apoio dos colegas $(r=0,43, p<0,01)$. A satisfação apresenta ainda correlações elevadas, mas negativas, com as práticas de formação $(r=-0,57, p<0,01)$, a comunicação organizacional $(r=-0,47, p<0,01)$ e a avaliação de desempenho $(r=-0,47, p<0,01)$. Da relação entre as medidas de saúde e as de clima, destacamos a correlação negativa e elevada entre bem-estar afectivo e sobrecarga de papel $(r=-0,44, p<0,01)$, as correlações negativas e moderadas entre funcionamento social e sobrecarga de papel $(r=-0,31, p<0,01)$ e conflito de papel $(r=-0,28, p<0,01)$, entre vitalidade e sobrecarga de papel $(r=-0,27, p<0,01)$ e conflito de papel $(r=-0,24, p<0,01)$, e entre funcionamento cognitivo e sobrecarga de papel $(r=-0,30, p<0,01)$. Salientamos ainda as correlações positivas e moderadas entre saúde física e latitude de decisão $(r=0,24, p<0,01)$, entre vitalidade e apoio dos colegas $(r=0,20, p<0,01)$, entre bem-estar afectivo e apoio do supervisor $(r=0,24, p<0,01)$ e entre funcionamento social e apoio do supervisor $(r=0,21, p<0,01)$. 
Tabela 1. Médias, desvios-padrão e correlações entre as variáveis de clima e de saúde e bem-estar

\begin{tabular}{|c|c|c|c|c|c|c|c|c|c|c|c|c|}
\hline & $M$ & $D P$ & 1 & 2 & 3 & 4 & 5 & 6 & 7 & 8 & 9 & 10 \\
\hline 1. Idade & 38,03 & 11,63 & & & & & & & & & & \\
\hline 2. Função & & & $-0,056$ & & & & & & & & & \\
\hline 3. Latitude decisão & 3,07 & 0,94 & $-0,012$ & $0,301^{\star \star}$ & $(0,78)$ & & & & & & & \\
\hline 4. Sobrecarga papel & 2,41 & 0,86 & 0,018 & $0,151^{\star \star}$ & $0,144^{\star \star}$ & $(0,78)$ & & & & & & \\
\hline 5. Clareza papel & 4,12 & 0,76 & $0,138^{\star *}$ & 0,025 & $0,342^{\star \star}$ & 0,015 & $(0,82)$ & & & & & \\
\hline 6. Conflito papel & 1,95 & 0,74 & $-0,061$ & $-0,022$ & $-0,074$ & $0,353^{* *}$ & $-0,119^{\star}$ & $(0,69)$ & & & & \\
\hline 7. Feedback desempenho & 2,32 & 0,58 & 0,075 & $-0,143^{\star \star}$ & $-0,103$ & $0,106^{\star}$ & $-0,231^{\star \star}$ & $0,243^{\star \star}$ & $(0,66)$ & & & \\
\hline 8. Apoio supervisor & 2,93 & 1,14 & $-0,026$ & $-0,040$ & $0,116^{*}$ & $-0,080$ & $0,185^{\star \star}$ & $-0,128^{*}$ & $-0,115^{\star}$ & $(0,90)$ & & \\
\hline 9. Liderança participativa & 2,31 & 1,00 & 0,098 & $0,188^{\star \star}$ & $0,483^{* \star}$ & 0,058 & $0,201^{\star \star}$ & $-0,082$ & $-0,146^{\star \star}$ & $0,432^{\star \star}$ & $(0,83)$ & \\
\hline 10. Apoio colegas & 3,15 & 1,22 & $-0,186^{\star \star}$ & $0,118^{\star}$ & $0,210^{\star \star}$ & $-0,060$ & $0,115^{*}$ & $-0,202^{\star \star}$ & $-0,150^{\star \star}$ & $0,375^{\star \star}$ & $0,322^{\star \star}$ & $(0,92)$ \\
\hline 11. Resposta mudança & 3,61 & 0,73 & $-0,044$ & $0,254^{\star \star}$ & $0,119^{\star}$ & $0,192^{\star \star}$ & $-0,064$ & 0,080 & 0,011 & $-0,151^{\star \star}$ & $-0,007$ & $-0,092$ \\
\hline 12. Avaliação desempenho & 3,08 & 0,71 & 0,101 & $0,109^{\star}$ & $-0,029$ & $0,137^{\star \star}$ & $-0,171^{\star \star}$ & $0,132^{\star}$ & $0,226^{\star \star}$ & $-0,233^{\star \star}$ & $-0,176^{\star \star}$ & $-0,210^{\star \star}$ \\
\hline 13. Liberdade & 2,80 & 0,63 & $-0,088$ & $-0,053$ &,$- 208^{\star \star}$ & 0,060 & $-0,176^{\star \star}$ & $0,140^{\star \star}$ & 0,073 & $-0,173^{* \star}$ & $-0,184^{\star \star}$ & $-0,147^{\star \star}$ \\
\hline 14. Cooperação e conflito & 3,34 & 0,65 & $-0,042$ & 0,083 & $-0,033$ & $0,226^{* *}$ & $-0,118^{*}$ & $0,197^{\star \star}$ & $0,178^{\star *}$ & $-0,172^{\star \star}$ & $-0,116^{\star}$ & $-0,317^{\star \star}$ \\
\hline 15. Formação & 2,95 & 0,66 & 0,083 & $0,190^{\star \star}$ & 0,001 & $0,228^{* \star}$ & $-0,075$ & $0,185^{\star \star}$ & $0,164^{\star \star}$ & $-0,295^{* \star}$ & $-0,169^{\star \star}$ & $-0,265^{\star \star}$ \\
\hline 16. Distribuição recursos & 3,47 & 0,65 & 0,025 & 0,071 & $-0,099$ & $0,313^{* \star}$ & $-0,018$ & $0,199^{\star \star}$ & $0,110^{*}$ & $-0,102$ & $-0,124^{\star}$ & $-0,130^{\star}$ \\
\hline 17. Comunicação & 3,36 & 0,70 & 0,022 & $0,268^{* \star}$ & 0,070 & $0,188^{* *}$ & $-0,091$ & 0,094 & $0,108^{*}$ & $-0,237^{\star *}$ & $-0,073$ & $-0,147^{\star \star}$ \\
\hline 18. Bem-estar afectivo & 4,58 & 0,93 & $-0,118^{\star}$ & $-0,219^{\star \star}$ & 0,038 & $-0,442^{\star \star}$ & $0,114^{\star}$ & $-0,246^{\star \star}$ & $-0,151^{\star \star}$ & $0,239^{\star \star}$ & 0,061 & $0,187^{\star \star}$ \\
\hline 19. Saúde física & 3,63 & 0,81 & $-0,164^{\star \star}$ & $0,107^{\star}$ & $0,235^{\star \star}$ & $-0,103$ & 0,076 & $-0,179^{\star *}$ & $-0,150^{* \star}$ & $0,127^{*}$ & $0,157^{\star \star}$ & $0,162^{* *}$ \\
\hline 20. Funcionamento social & 2,02 & 0,34 & $-0,044$ & $-0,022$ & 0,072 & $-0,307^{\star \star}$ & 0,075 &,$- 275^{\star *}$ & $-0,141^{* \star}$ & $0,210^{\star \star}$ & 0,099 & $0,195^{\star *}$ \\
\hline 21. Vitalidade & 3,78 & 0,97 & $-0,052$ & 0,009 & $0,151^{\star \star}$ & $-0,274^{\star \star}$ & 0,049 & $-0,244^{\star \star}$ & $-0,111^{\star}$ & $0,130^{*}$ & $0,191^{\star \star}$ & $0,200^{\star *}$ \\
\hline 22. Funcionamento cognitivo & 2,99 & 0,43 & 0,010 & $-0,161^{\star \star}$ & $0,118^{*}$ & $-0,301^{* \star}$ & 0,048 & $-0,140^{\star *}$ & $-0,114^{*}$ & 0,076 & 0,094 & $0,110^{*}$ \\
\hline 23. Satisfação & 4,05 & 1,02 & $-0,104$ & $-0,098$ & $0,199^{\star *}$ & $-0,262^{\star *}$ & $0,141^{\star \star}$ & $-0,229^{\star \star}$ & $-0,109^{\star}$ & $0,491^{\star *}$ & $0,365^{\star \star}$ & $0,430^{\star \star}$ \\
\hline
\end{tabular}

(Cont.)

\begin{tabular}{|c|c|c|c|c|c|c|c|c|c|c|c|c|}
\hline & 11 & 12 & 13 & 14 & 15 & 16 & 17 & 18 & 19 & 20 & 21 & 22 \\
\hline \multicolumn{13}{|l|}{ 1. Idade } \\
\hline \multicolumn{13}{|l|}{ 2. Função } \\
\hline \multicolumn{13}{|l|}{ 3. Latitude decisão } \\
\hline \multicolumn{13}{|l|}{ 4. Sobrecarga papel } \\
\hline \multicolumn{13}{|l|}{ 5. Clareza papel } \\
\hline \multicolumn{13}{|l|}{ 6. Conflito papel } \\
\hline \multicolumn{13}{|l|}{ 7. Feedback desempenho } \\
\hline \multicolumn{13}{|l|}{ 8. Apoio supervisor } \\
\hline \multicolumn{13}{|l|}{ 9. Liderança participativa } \\
\hline \multicolumn{13}{|l|}{ 10. Apoio colegas } \\
\hline 11. Resposta mudança & $(0,93)$ & & & & & & & & & & & \\
\hline 12. Avaliação desempenho & $0,324^{\star \star}$ & $(0,71)$ & & & & & & & & & & \\
\hline 13. Liberdade & $0,112^{\star}$ & 0,091 & $(0,73)$ & & & & & & & & & \\
\hline 14. Cooperação e conflito & $0,435^{\star \star}$ & $0,397^{\star \star}$ & $0,216^{\star \star}$ & $(0,80)$ & & & & & & & & \\
\hline 15. Formação & $0,521^{\star \star}$ & $0,496^{\star \star}$ & $0,125^{*}$ & $0,463^{\star \star}$ & $(0,74)$ & & & & & & & \\
\hline 16. Distribuição recursos & $0,333^{\star \star}$ & $0,327^{\star \star}$ & 0,084 & $0,327^{\star \star}$ & $0,403^{\star \star}$ & $(0,74)$ & & & & & & \\
\hline 17. Comunicação & $0,574^{\star \star}$ & $0,480^{\star \star}$ & $0,173^{\star \star}$ & $0,542^{\star \star}$ & $0,549^{\star \star}$ & $0,359^{\star \star}$ & $(0,89)$ & & & & & \\
\hline 18. Bem-estar afectivo & $-0,240^{\star *}$ & $-0,209^{\star \star}$ & $-0,186^{\star *}$ & $-0,292^{\star *}$ & $-0,321^{* *}$ & $-0,257^{* *}$ & $-0,284^{* *}$ & $(0,91)$ & & & & \\
\hline 19. Saúde física & 0,026 & 0,041 & $-0,158^{\star *}$ & $-0,018$ & $-0,031$ & $-0,040$ & 0,018 & $0,367^{\star \star}$ & $(0,77)$ & & & \\
\hline 20. Funcionamento social & $-0,122^{\star}$ & $-0,152^{\star \star}$ & $-0,157^{\star *}$ & $-0,264^{\star *}$ & $-0,203^{* *}$ & $-0,172^{* \star}$ & $-0,149^{* \star}$ & $0,519^{\star *}$ & $0,461^{* *}$ & $(0,83)$ & & \\
\hline 21. Vitalidade & $-0,151^{\star \star}$ & $-0,111^{\star}$ & $-0,111^{*}$ & $-0,204^{\star *}$ & $-0,162^{* \star}$ & $-0,138^{* *}$ & $-0,065$ & $0,463^{\star \star}$ & $0,459^{\star \star}$ & $0,543^{\star \star}$ & $(0,83)$ & \\
\hline 22. Funcionamento cognitivo & $-0,193^{\star *}$ & $-0,195^{\star \star}$ & $-0,142^{\star *}$ & $-0,222^{\star \star}$ & $-0,212^{* \star}$ & $-0,186^{* \star}$ & $-0,286^{* *}$ & $0,425^{\star *}$ & $0,228^{\star *}$ & $0,405^{* *}$ & $0,310^{\star \star}$ & $(0,78)$ \\
\hline 23. Satisfação & $-0,450^{\star *}$ & $-0,473^{\star \star}$ & $-0,258^{* *}$ & $-0,441^{* *}$ & $-0,568^{* *}$ & $-0,423^{* *}$ & $-0,468^{* *}$ & $0,426^{* \star}$ & $0,207^{* \star}$ & $0,321^{* *}$ & $0,296^{\star \star}$ & $0,287^{\star \star}$ \\
\hline
\end{tabular}

Nota: ${ }^{* *} p<0,01 ; * \mathrm{p}<0,05$. Entre parêntesis encontram-se os valores de $\alpha$ de cronbach 


\section{Teste de hipóteses}

Para avaliarmos as associações previstas na primeira hipótese, entre as facetas de clima psicológico e dimensões da saúde, fomos realizar análises de regressão separadas para cada uma das cinco dimensões da saúde. As quinze facetas do clima psicológico foram introduzidas como variáveis independentes em cada uma das regressões, depois de controlar o efeito da idade e da categoria profissional ${ }^{2}$.

Como podemos ver na Tabela 2, as facetas do clima em conjunto explicam uma parte significativa da variância nas cinco dimensões de saúde, a qual varia entre 16 por cento da saúde física $\left(R^{2}=0,157, p<0,01\right)$ e os 33 por cento do bem-estar afectivo $\left(R^{2}=0,325, p<0,01\right)$. Estes resultados suportam claramente a primeira hipótese.

O mesmo procedimento foi seguido para testarmos a segunda hipótese, que prevê a associação entre as facetas do clima e a satisfação. A análise de regressão da satisfação nas facetas do clima mostra que estas explicam, em conjunto 62 por cento da variância na satisfação $\left(R^{2}=0,617, p<0,01\right)$. Estes resultados vêm claramente em suporte da nossa segunda hipótese.

No entanto, como podemos ver na análise das correlações (Tabela 1), as facetas do clima não têm todas a mesma força, na associação que estabelecem quer com a saúde, quer com a satisfação. Mais ainda, algumas facetas parecem ser promotoras da saúde, ao estarem positivamente associadas com a saúde, enquanto outras aparecem negativamente associadas com a saúde, indicando que a sua presença pode ser prejudicial para a saúde dos participantes. Pareceu-nos, então, que deveríamos procurar tornar mais clara a relação entre as diferentes facetas do clima e a saúde e satisfação. Para tal, fomos estimar a importância relativa das facetas de clima enquanto preditores da saúde, seguindo o procedimento proposto por Johnson e LeBreton (2004). O principal objectivo desta análise é compreender o contributo unico de cada faceta do clima para a saúde e satisfação dos participantes. Este tipo de análise tem, para além da relevância teórica associada ao controle da colinearidade em modelos mulitdimensionais (LeBreton, Ployhart \& Ladd, 2004), uma utilidade prática importante, na medida em que permite intervenções mais precisas e de maior validade. Este procedimento consiste em calcular o contributo de cada preditor, em termos da proporção do $R^{2}$ do modelo global que lhe pode ser atribuída (Johnson \& LeBreton, 2004).

\footnotetext{
${ }^{2}$ Os indivíduos com funções de estatuto inferior têm maior probabilidade de referir sintomas de perturbação da saúde, o que pode estar relacionado com o estatuto social (Jamison, Wallace \& Jamison, 2004) ou com o estatuto socioeconómico (Lynch, Krause, Kaplan, Tuomilehto \& Salonem, 1997).
} 
Tabela 2. Importância relativa das facetas de clima na predição da satisfação e da saúde

\begin{tabular}{|c|c|c|c|c|c|c|}
\hline & $\begin{array}{l}\text { Saúde } \\
\text { física }\end{array}$ & $\begin{array}{l}\text { Bem-estar } \\
\text { afectivo }\end{array}$ & Vitalidade & $\begin{array}{c}\text { Funcionamento } \\
\text { social }\end{array}$ & $\begin{array}{c}\text { Funcionamento } \\
\text { cognitivo }\end{array}$ & Satisfação \\
\hline & $\% R^{2}$ & $\% R^{2}$ & $\% R^{2}$ & $\% R^{2}$ & $\% R^{2}$ & $\% R^{2}$ \\
\hline Idade & 17,2 & 4,0 & 1,7 & 1,0 & 0,3 & 1,4 \\
\hline Função & 3,8 & 5,3 & 0,6 & 0,4 & 6,7 & 1,3 \\
\hline Resposta à Mudança & 0,2 & 3,3 & 7,0 & 1,2 & 4,5 & 9,2 \\
\hline Comunicação & 0,6 & 4,6 & 2,1 & 1,6 & 16,1 & 6,3 \\
\hline Conflito e Cooperação & 0,6 & 5,7 & 8,0 & 13,2 & 4,6 & 5,7 \\
\hline Avaliação Desempenho & 4,4 & 1,5 & 1,0 & 1,8 & 4,5 & 9,1 \\
\hline Formação & 0,5 & 6,6 & 2,5 & 3,7 & 3,5 & 12,3 \\
\hline Liberdade & 9,6 & 4,7 & 1,8 & 4,9 & 4,1 & 3,2 \\
\hline Distribuição Recursos & 0,4 & 4,3 & 1,6 & 2,7 & 3,0 & 7,0 \\
\hline Apoio Colegas & 5,2 & 2,3 & 6,9 & 5,0 & 1,5 & 9,0 \\
\hline Apoio Supervisor & 4,1 & 6,9 & 1,8 & 10,0 & 0,4 & 14,9 \\
\hline Liderança participativa & 5,8 & 0,6 & 9,8 & 1,0 & 1,6 & 6,7 \\
\hline Feedback Desempenho & 7,5 & 2,1 & 1,6 & 2,9 & 2,7 & 0,3 \\
\hline Latitude de Decisão & 20,2 & 1,1 & 8,0 & 1,5 & 9,9 & 3,2 \\
\hline Clareza do Papel & 1,4 & 1,4 & 0,4 & 0,5 & 0,5 & 0,5 \\
\hline Conflito do Papel & 11,6 & 5,6 & 14,9 & 16,3 & 2,6 & 1,5 \\
\hline Sobrecarga de Papel & 6,1 & 35,4 & 27,8 & 27,3 & 31,1 & 2,9 \\
\hline R-quadrado & $0,157 * *$ & $0,325^{* *}$ & $0,186^{* *}$ & $0,193^{* *}$ & $0,200^{* *}$ & $0,618^{* *}$ \\
\hline
\end{tabular}

Nota: $* * p<0,01$ 
Os resultados apresentados na Tabela 2 sugerem que os principais preditores da vitalidade são a sobrecarga de trabalho $\left(28 \%\right.$ do $R^{2}$ total $)$, o conflito de papel (15\% do $R^{2}$ total) e a liderança participativa $\left(10 \%\right.$ do $R^{2}$ total). O funcionamento social é predito, em primeiro lugar, pela sobrecarga de trabalho ( $27 \%$ do $R^{2}$ total), seguida do conflito de papel ( $16 \%$ do $R^{2}$ total) e da cooperação na organização ( $13 \%$ do $R^{2}$ total). Os principais preditores da saúde física são a latitude de decisão ( $20 \%$ do $R^{2}$ total), o conflito de papel ( $12 \%$ do $R^{2}$ total) e a liberdade ( $10 \%$ do $R^{2}$ total). De salientar que a idade é responsável por 17 por cento da variância na saúde física. Os principais preditores do funcionamento cognitivo são a sobrecarga de trabalho (21\% do $R^{2}$ total), a comunicação organizacional $\left(16 \%\right.$ do $R^{2}$ total) e a latitude de decisão ( $10 \%$ do $R^{2}$ total). O bem-estar afectivo é predito sobretudo pela sobrecarga de trabalho (35\% do $R^{2}$ total), seguido do apoio do supervisor ( $7 \%$ do $R^{2}$ total) e das práticas de formação $\left(7 \%\right.$ do $R^{2}$ total). Finalmente, entre os principais preditores da satisfação aparecem o apoio do supervisor ( $15 \%$ do $R^{2}$ total), as práticas de formação (13\% do $R^{2}$ total), as práticas de avaliação de desempenho, a resposta à mudança e o apoio dos colaboradores todos com 9 por cento do $R^{2}$ total. Em resumo, a análise da importância relativa dos preditores veio dar suporte à primeira e segunda hipóteses, na medida em que demonstra que existe uma associação clara entre as percepções que os indíviduos têm de alguns aspectos do seu trabalho e de algumas práticas de recursos humanos da organização e a sua satisfação e a sua saúde avaliadas em dimensões distintas e complementares.

No que diz respeito à terceira hipótese, que previa que a relação entre clima psicológico e saúde era mediada pela satisfação com o trabalho e a organização, ela foi testada por meio de um conjunto de regressões hierárquicas. Esta análise consiste em contrastar a variância total e única explicada pelo conjunto de variáveis preditoras. De acordo com Cohen e Cohen (1983), este tipo de análise determina a proporção de variância independente explicada pelo conjunto de preditores (efeito total) e a proporção da variância explicada por cada um dos conjuntos de preditores com as outras variáveis controladas (efeito único). Efeitos significativos, quer totais, quer únicos, dos mediadores, assim como efeitos totais significativos, mas sem efeitos significativos únicos dos antecedentes, suportam o modelo de mediação. A determinação dos efeitos únicos e dos efeitos totais do conjunto de preditores é dada examinando os valores de $R^{2}$. 


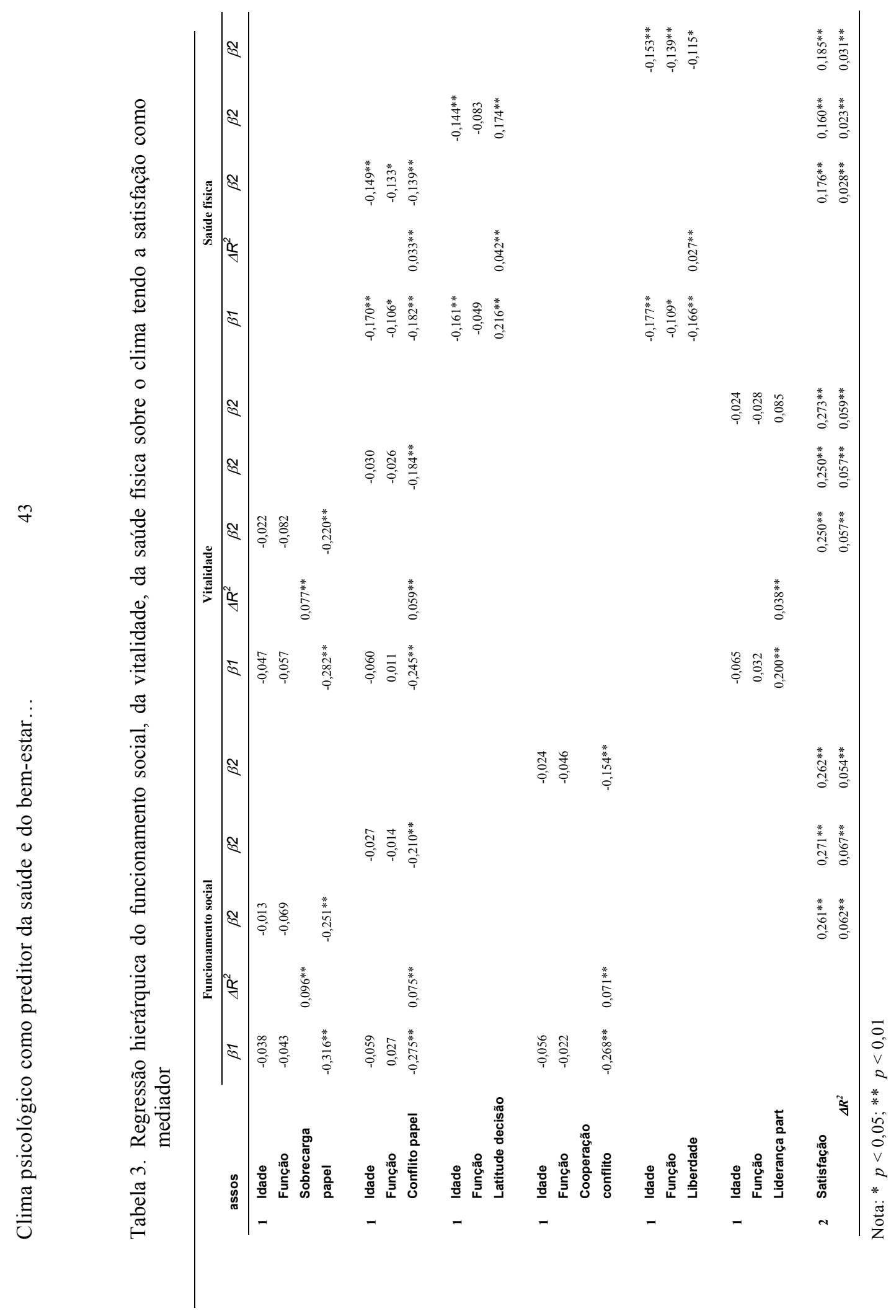


Tabela 4. Regressão hierárquica do bem-estar afectivo e do funcionamento cognitivo sobre o clima, tendo a satisfação como mediador

\begin{tabular}{|c|c|c|c|c|c|c|c|c|c|c|c|}
\hline \multirow{2}{*}{\multicolumn{2}{|c|}{ Passos }} & \multicolumn{5}{|c|}{ Bem-estar afectivo } & \multicolumn{5}{|c|}{ Funcionamento cognitivo } \\
\hline & & $\beta 1$ & $\Delta R^{2}$ & $\beta 2$ & $\beta 2$ & $\beta 2$ & $\beta 1$ & $\Delta R^{2}$ & $\beta 2$ & $\beta 2$ & $\beta 2$ \\
\hline \multirow[t]{3}{*}{1} & Idade & $-0,113^{*}$ & & $-0,082$ & & & $-0,014$ & & $-0,035$ & & \\
\hline & Função & $0,105^{\star}$ & & 0,071 & & & $-0,091$ & & $-0,067$ & & \\
\hline & Sobrecarga papel & $-0,424^{* *}$ & $0,174^{\star \star}$ & $-0,346^{* *}$ & & & $0,285^{\star *}$ & $0,079^{\star \star}$ & $0,231^{* *}$ & & \\
\hline \multirow[t]{3}{*}{1} & Idade & $-0,116^{*}$ & & & $-0,081$ & & & & & & \\
\hline & Função & $0,170^{\star *}$ & & & $0,118^{*}$ & & & & & & \\
\hline & Apoio supervisor & $0,230^{\star *}$ & $0,053^{* *}$ & & 0,044 & & & & & & \\
\hline \multirow[t]{3}{*}{1} & Idade & $-0,094$ & & & & $-0,075$ & & & & & \\
\hline & Função & $0,114^{*}$ & & & & $0,106^{*}$ & & & & & \\
\hline & Formação & $-0,288^{* *}$ & $0,078^{\star *}$ & & & $-0,088$ & & & & & \\
\hline \multirow[t]{3}{*}{1} & Idade & & & & & & $-0,008$ & & & $-0,034$ & \\
\hline & Função & & & & & & $-0,200^{* *}$ & & & $-0,147^{\star *}$ & \\
\hline & Latitude decisão & & & & & & $-0,176^{* *}$ & $0,028^{\star \star}$ & & $-0,113^{*}$ & \\
\hline \multirow[t]{3}{*}{1} & Idade & & & & & & $-0,018$ & & & & $-0,034$ \\
\hline & Função & & & & & & $-0,070$ & & & & $-0,065$ \\
\hline & Comunicação & & & & & & $0,265^{\star *}$ & $0,065^{\star \star}$ & & & $0,174^{\star *}$ \\
\hline \multirow[t]{2}{*}{2} & Satisfação & & & $0,322^{* *}$ & $0,385^{\star *}$ & $0,357^{* *}$ & & & $-0,222^{* *}$ & $-0,239^{* *}$ & $-0,200^{* *}$ \\
\hline & $\Delta R^{2}$ & & & $0,094^{* *}$ & $0,109^{\star \star}$ & $0,086^{\star *}$ & & & $0,045^{\star \star}$ & $0,052^{\star *}$ & $0,031^{* *}$ \\
\hline
\end{tabular}

Nota: $* p<0,05, * * p<0,01$

Como vimos na análise anterior, as facetas do clima não têm todas a mesma importância na predição das dimensões da saúde, tendo muitos delas uma associação mesmo insignificante. Por exemplo, a liderança participativa e a latitude de decisão têm um efeito negligenciável no bem-estar afec- 
tivo, 0,6 e 1,1 do $R^{2}$ total, respectivamente. Mais ainda, Cohen e Cohen (1983) estabelecem que, para haver mediação, as três variáveis devem estar significativamente correlacionadas. Tendo isto em consideração, optámos por testar os efeitos de mediação da satisfação apenas para os três preditores mais importantes de cada uma das cinco dimensões da saúde, obtidos através da análise da importância relativa descrita anteriormente (Johnson \& LeBreton, 2004).

Os resultados destas análises de regressão suportam, em parte, a nossa terceira hipótese, segundo a qual o efeito das facetas de clima na saúde dos profissionais pode ser mediado pela sua satisfação com o trabalho e a organização (Tabelas 3 e 4). Em relação às distintas dimensões da saúde, verificámos que os efeitos da latitude de decisão, do conflito de papel e da autonomia na saúde física são apenas parcialmente mediadas pela satisfação. Do mesmo modo, os efeitos da sobrecarga de trabalho, do conflito de papel, e da cooperação no funcionamento social são parcialmente mediados pela satisfação. Os efeitos da sobrecarga de trabalho e do conflito de papel na vitalidade são também parcialmente mediados pela satisfação, enquanto o efeito da liderança participativa na vitalidade é completamente mediado pela satisfação. No que diz respeito ao funcionamento cognitivo, os efeitos da sobrecarga de trabalho, da latitude de decisão e da comunicação são parcialmente mediados pela satisfação. Por fim, os efeitos da sobrecarga de trabalho no bem-estar afectivo são parcialmente mediados pela satisfação, enquanto os efeitos do apoio do supervisor e das práticas de formação são completamente mediados pela satisfação. Estes resultados parecem apontar para a ideia de que a satisfação com certas características do papel, com o ambiente social, com a acção do supervisor ou com as práticas organizacionais pode ter um efeito positivo na saúde dos trabalhadores, mas que, para além desse, existe um efeito directo, geralmente negativo, de certas características do trabalho e da organização na saúde dos indivíduos. No próximo ponto, discutiremos em detalhe as consequências teóricas e práticas destes resultados.

\section{Discussão}

Este estudo teve como objectivo analisar a influência das percepções do ambiente de trabalho sobre a auto-avaliação da saúde.

A influência das caracaterísticas do ambiente de trabalho na saúde é consensual. No entanto, os estudos que consideram estas características como sendo percepcionadas e interpretadas enquanto atributos de uma realidade socialmente construída são escassos (Parker et al., 2003). 
O ambiente de trabalho, nesta perspectiva, pode ser analisado tendo em conta um conjunto mais restrito ou mais alargado de aspectos que tente dar conta da complexidade na qual decorre a actividade do trabalhador. Esta abordagem global, molar ou de banda larga tem sido defendida por alguns autores como a via mais adequada quando se pretende analisar os preditores de fenómenos mais globais (Carr et al., 2003), como a presença de bem-estar físico, psicológico e social. A abordagem multidimensional é mais vantajosa quando se pretende uma visão global da forma como a organização funciona como um todo (Ashkanasy, Wilderon \& Peterson, 2000; Patterson, West, Shackleton, Dawson, Lawthorn, Maitlis, Robinson \& Wallace, 2005).

Neste estudo, optou-se por um conjunto alargado de aspectos que, tendo como fio condutor os referentes situacionais propostos por Jones e James (1979), permitissem considerar, simultaneamente e sem ambiguidade, no quadro de referência, aspectos directamente associados aos individuos como as características do papel e o apoio de colegas e supervisor. e indirectos, como as práticas organizacionais de formação e de resposta à umdança. Esta abordagem tenta minimizar o risco dos respondentes descreverem percepções sobre partes diferentes da organização, porque uns assumem que o questionário se refere ao seu departamento, enquanto outros assumem que se refere à organização (Rousseau, 1988).

Tendo abordado o clima psicológico de acordo com cinco dimensões, que foram avaliadas através de dezasseis facetas, tivemos a oportunidade de analisar a importância de cada uma na explicação da auto-avaliação da saúde desta amostra de profissionais de saúde.

A saúde relacionada com o trabalho tem sido operacionalizada de muitas formas parciais. Neste estudo, explorou-se uma operacionalização do conceito definido pela Organização Mundial de Saúde (WHO, 1948) que considera a saúde como a presença de bem-estar físico, psicológico e social.

Tendo presente esta operacionalização do clima psicológico e da saúde relacionada com o trabalho, fomos testar primeiramente a hipótese de que quanto mais positivas forem as percepções sobre o ambiente de trabalho melhor será a saúde, isto é, mais elevados serão os níveis de vitalidade, funcionamento social e cognitivo, saúde física e psicológica.

Os dados obtidos, através da análise das correlações e das regressões, suportam claramente esta hipótese. A variância explicada de cada dimensão da saúde varia entre os dezasseis e os trinta e três por cento.

Mais, é possível perceber que as facetas do clima não têm todas a mesma força de associação na relação que estabelecem com a saúde. Alguns factores, como, por exemplo, o apoio de colegas e supervisor, latitude de decisão e práticas de formação, aparecem como promotores da saúde ao estarem positivamente associados. Outros, como, por exemplo, a sobrecarga e o conflito de papel, ao estarem negativamente associados, aparecem como 
factores de risco, indicando que a sua presença pode ser prejudicial para a saúde.

Estes dados são suportados quer pela literatura sobre stress no trabalho, quer na que se debruça sobre o desenho do trabalho. Nomeadamente, os estudos sobre as exigências do trabalho no disfuncionamento e patologia dos profissionais de saúde, bem como os estudos que identificam os ganhos associados quer à diminuição das exigências, nomeadamente, na melhoria da exaustão emocional, como o estudo longitudinal desenvolvido por Houkes, Janssen, De Jonge e Bakker (2003), quer na diminuição das exigências objectivas, como o estudo com examinadores de condução, de Parkes (1995), em que a redução objectiva da sobrecarga de trabalho teve como resultado a redução dos níveis de ansiedade, aumento da satisfação com o trabalho e melhoria do desempenho cognitivo.

Estes dados também são suportados pela investigação sobre o efeito promotor na saúde, do suporte social (Viswervaran, Sanchex \& Fisher, 1999), assim como a investigação sobre o enriquecimento do trabalho, que advoga, entre outras medidas, o aumento da latitude de decisão e das práticas de formação. Os resultados dos estudos transversais e longitudinais sobre o desenho do trabalho convergem na noção de que os trabalhos enriquecidos ou a presença de características do trabalho como a autonomia ou o uso de competências são promotores da satisfação (Wall, Kemp, Jackson \& Clegg, 1986). Por exemplo, Parker, Chmiel e Wall (1997) demonstraram que o aumento da clareza do papel e a participação na tomada de decisão estavam associados com a melhoria da saúde, durante um periodo de downsizing.

Suportando esta hipótese, sublinha-se ainda as relações diferenciadas dos preditores com várias dimensões da saúde, por exemplo, a sobrecarga de trabalho que é o melhor preditor da vitalidade, funcionamento cognitivo, social e bem-estar psicológico, mas não do bem-estar físico, o que pode estar associado com a natureza das actividades de saúde que são mais exigentes a nível emocional e cognitivo do que físico.

A segunda hipótese, que propõe que quanto mais positivas forem as percepções sobre o trabalho mais positiva será a satisfação, também é suportada porque todas as dimensões do clima se correlacionam significativamente com a satisfação, algumas de forma positiva e outras de forma negativa. Estes dados posicionam-se no mesmo sentido dos dados dos estudos de Spector (1997) e de Jonge et al. (2001), que estudaram longitudinalmente profissionais de saúde. A análise de regressão da satisfação nas facetas do clima mostra que estas explicam em conjunto sessenta e dois por cento da variância da satisfação, sendo o apoio do supervisor e dos colaboradores, as práticas de formação e de avaliação do desempenho e a resposta da organização à mudança os melhores preditores. Estes dados apontam 
para as potencialidades de intervenção do desenho do trabalho, nomeadamente na melhoria das práticas organizacionais.

Tendo em conta que a satisfação tem sido considerada como um factor importante na explicação de atitudes e comportamentos relacionados com o trabalho (Kopelman, Brief \& Guzzo, 1990), pusemos como terceira hipótese, que a satisfação poderia ser um mediador da relação entre clima psicológico e saúde. Os dados suportam parcialmente esta hipótese, porque, em regra, todas as relações são parcialmente mediadas pela satisfação com a excepção do apoio do supervisor e das práticas de formação no bem-estar psicológico e a liderança participativa na vitalidade, cujo efeito é completamente mediado pela satisfação.

Deste estudo ressalta a importância da satisfação na forma como as percepções sobre o ambiente de trabalho influenciam a saúde destes profissionais de saúde. Atendendo a que as práticas organizacionais de formação, avaliação de desempenho e de resposta à mudança, bem como o suporte social de superiores e colegas, são os principais preditores da saúde, sublinham-se as intervenções com foco nas práticas da organização, bem como na melhoria do ambiente social, que visam aumentar a satisfação. Kopelman (1985), numa análise de 32 estudos de intervenção com desenho do trabalho, refere que a satisfação com o trabalho aumentou em $80 \%$ destes estudos. A evidência, obtida longitudinalmente, sugere, em geral, que o aumento da satisfação com o trabalho conduz ao aumento do bem-estar. Por exemplo Winefield, Tiggemann e Goldney (1998) realizaram um grande estudo longitudinal com jovens, no qual o aumento da satisfação estava relacionado com a saúde psicológica ao longo do tempo. Por outro lado, as intervemções que aumentam a satisfação também aumentam o bem-estar ao longo do tempo (Barrios-Choplin, McCraty \& Cryer, 1997) e as emoções positivas parecem proteger os indivíduos do stress (Folkman \& Moskowitz, 2000).

Como argumento final, sublinha-se que a análise do impacto das intervenções de gestão de stress no bem-estar tem demonstrado a existência de um efeito multiplicativo no tempo, porque o aumento do bem-estar e a diminuição dos stressores influenciam-se mutuamente (Daniels \& Guppy, 1997; Edwards, 1998).

\section{Conclusões}

Os dados desta investigação suportam a hipótese de que as percepções dos profissionais de saúde desta amostra, sobre o ambiente de trabalho, influenciam a sua satisfação e saúde. Mais, estas percepções vão exercer uma influência directa, quer na saúde, quer na satisfação, e indirecta, na saúde via satisfação. 
Este estudo tem várias contribuições. Primeiro, suporta a utilização de uma perspectiva global do clima psicológico, por ser mais dêscriminativa, para a análise de fenómenos complexos, como, por exemplo, a saúde e o bem-estar relacionados com o trabalho. Adicionalmente, sugere que esta pode ser uma via de diagnóstico que permite desenvolver e implementar estratégias de saúde ocupacional nomeadamente no âmbito da gestão do stress (Nelson \& Simmons, 2003) e do desenho do trabalho e, em última instância, de criação de organizações saudáveis (Parker, Turner \& Griffin, 2003).

Segundo, avança com uma operacionalização da saúde relacionada com o trabalho enquanto funcionamento físico, psicológico e social (WHO, 1948), mas também cognitivo e com vitalidade (Keyes, 2005; Warr, 1990a).

Terceiro, vem dar suporte à importância dos diagnósticos de clima nas organizações, que, sendo uma prática corrente, não são, em regra, considerados enquanto excelente fonte de informação para delinear estratégias de intervenção na saúde ocupacional.

Quarto, apesar de o bem-estar psicológico ter sido frequentemente operacionalizado como satisfação no trabalho (Clegg \& Wall, 1981), estes fenómenos são distintos, embora relacionados. Por outro lado, a perspectiva dominante na conceptualização do clima psicológico, ou seja, das percepções partilhadas sobre os acontecimentos, práticas e procedimentos da organização, tem considerado estas percepções como descritivas mais do que afectivas ou avaliativas (Schneider \& Reichers, 1983). Os dados deste estudo sugerem a existência de uma componente avaliativa ou afectiva forte, que também é sugerida por Patterson, Warr e West (2004).

Este estudo apresenta várias limitações. Primeiro, os dados foram recolhidos num único momento e analisados através de correlações e regressões. Como resultado, não podemos inferir relações causais entre as variáveis estudadas. Por exemplo, é possível que as pessoas saudáveis tenham percepções mais positivas sobre o ambiente de trabalho ou que as pessoas menos saudáveis tenham desistido do seu trabalho (Virtanen, Liukkonen, Virtanen, Vahtera \& Ferrieg, 2005). Em estudos futuros, existe a necessidade de desenvolver estudos longitudinais (Zapf, Dormann \& Frese, 1996; Schmidt, 1996) para, especificamente, analisar a dinâmica do processo subjácente à influência das percepções sobre a saúde (Garst, Frese \& Molenaar, 2000).

Segundo, pode-se considerar uma limitação o número de variáveis usadas neste estudo, mas, como referem Cohen e Cohen (1983), quando se reduzem variáveis aumenta-se o poder explicativo, mas o que se ganha em poder de teste, perde-se em especificidade. Nesta amostra, por exemplo, das várias práticas da organização só a formação, a avaliação de desempenho e o conflito e cooperação é que aparecem como preditoras da saúde. Este tipo de opção é mais útil quando se tem como objectivo o diagnóstico para pos- 
terior intervenção na mudança. Por outro lado, tivemos em conta o número de variáveis preditoras e os potenciais efeitos de colinearidade associados e tentámos minimizá-los, usando um procedimento estatístico desenvolvido por Johnson e LeBreton (2004) que permite controlar este efeito.

Terceiro, o uso exclusivo de dados auto-referidos levanta os problemas da variância do método comum, por exemplo, o risco de inflação das correlações (Kasl, 1986; Kessler, 1987) bem como a distorção das verdadeiras associações (Williams \& Brown, 1994). Apesar de Spector e Brannick (1995) terem demonstrado que este erro era mais a excepção do que a regra, e de, neste estudo, ele poder ter sido diminuído pela utilização de diferentes escalas de resposta, pela utilização de itens invertidos, pela utilização de diferentes dimensões em cada escala e pelas análises factoriais, que revelaram que as facetas do clima correspondiam a factores independentes, consideramos que, no futuro, é necessário utilizar uma combinação de métodos (Bailey \& Bhagat, 1987). Esta metodologia permitirá associar aos dados de auto-avaliação os dados provenientes da observação por terceiros, que também está sujeita a erros de medida e que, por isso, não é melhor nem mais "objectiva" (Eisenberg, 1979; Kaplan \& Camacho, 1983; Semmer, Grebner \& Elfering, 2004), mas proporciona informação valiosa que permite reduzir o efeito da variância comun.

\section{Referências}

Aasland, O. G., Olff, M., Schweder, T. \& Ursin, H. (1997). Health complaints and job stress in Norwegian physicians: the use of an overlapping questionnaire design. Social Science Medicine, 45, 1615-1625.

Ajzen, I. \& Fishbein, M. (1980). Understanding attitudes and predicting social behavior. Englewood Cliffs, NJ: Prentice Hall.

Almond, S. \& Healey, A. (2003). Mental health and absence from work: new evidence from the UK Quarterly Labour Force Survey. Work, Employment and Society, 17, 731-742.

Ashforth, B. E. (1985). Climate formation: issues and extensions. Academy of Management Review, 10, 837-847.

Ashkanasy, N. M., Wilderon, C. P. M. \& Peterson, M. F. (2000). Introduction. In N. M. Ashkanasy, C. P. M. Wilderom \& M. F. Peterson (Eds.), Handbook of organizational culture and climate (pp. 1-18). Thousand Oaks, CA: Sage Publications.

Bailey, J. M. \& Bhagat, R. S. (1987). Meaning and measurement of stressors in the work environment: an evaluation. In S. V. Kasl \& C. L. Cooper (Eds.), Stress and health: Issues in research methodology (pp. 207-229). Chichester, UK: Wiley. 
Barrios-Choplin, B., McCraty, R. \& Cryer, B. (1997). An inner quality approach to reducing stress and improving physical and emotional well-being at work. Stress Medicine, 13, 193-201.

Breaugh, J. A. \& Colihan, J. P. (1994). Measuring facets of job ambiguity: construct validity evidence. Journal of Applied Psychology, 79, 191-202.

Brooke, P. P., Russell, D. W. \& Price, J. L. (1988). Discriminant validation of measures of job satisfaction, job involvement, and organizational commitment. Journal of Applied Psychology, 73, 139-145.

Cameron, K. S. \& Caza, A. (2004). Contributions to the discipline of positive organizational scholarship. American Behavioral Scientist, 47, 1-9.

Caplan, R. (1994). Stress, anxiety and depression in hospital consultants, general practioners and senior health service managers. British Medical Journal, 309, 1261-1263.

Carr, J. Z., Schmidt, A. M., Ford, K. \& DeShon, R. P. (2003). Climate perceptions matter: a meta-analytic path analysis relating molar climate, cognitive and affective states, and individual level work outcomes. Journal of Applied Psychology, 88, 605-619.

Cass, M. H., Siu, O. L., Faragher, E. B. \& Cooper, C. L. (2003). A meta-analysis of the relationship between job satisfaction and employee health in Hong Kong. Stress and Health, 19, 79-95.

Chambel, M. J. \& Curral, L. (1998). Questionário de clima e satisfação organizacional. Faculdade de Psicologia e de Ciências da Educação, Universidade de Lisboa.

Clegg, C. \& Wall, T. D. (1981). A note on some new scales for measuring aspects of psychological well-being at work. Journal of Occupational Psychology, 54, 221-225.

Cronbach, L. J. \& Gleser, G. C. (1957). Psychological tests and personnel decisions. Champaign, IL: University of Illinois Press.

Daniels, K. \& Guppy, A. (1997). Stressors, locus de control, and social support as consequences of affective psychological well-being. Journal of Occupational Health Psychology, 2, 156-174.

Danna, K. \& Griffin, R. W. (1999). Health and well-being in the workplace: a review and siyntesis of the literature. Journal of Management, 25, 357-384.

Day, D. V. \& Bedeian, A. G. (1991). Work climate and type. A status as predictors of job satisfaction: a test of the interactional perspective. Journal of Vocational Behavior, 38, 39-52.

De Lange, A. H., Taris, T. W., Kompier, M., Houtman, I. \& Bongers, P. (2004). The relationship between work characteristics and mental health: Examining normal, reversed and reciprocal relationship in a 4-wave study. Work \& Stress, 18 , 149-166.

Diener, E., Suh, E. M., Lucas R. E. \& Smith, H. I. (1999). Subjective well-being: three decades of progress. Psychological Bulletin, 125, 267-302.

Eisenberg, J. M. (1979). Sociocultural influences on decision-making by clinicians. Annual International Medicine, 90, 949-956. 
European Union (EU) (2004). Work and health in the EU: a statistical portrait. Brussels: European Comission.

European Union (EU) (2005). Growth and jobs. Luxembourg: Office for Official Publications of the European Communities.

Folkman, S. \& Moskowitz, J. T. (2000). Positive affect and the other side of coping. American Psychologist, 55, 647-654.

Ganster, D. C. \& Schaubroeck, J. (1991). Work stress and employee health. Academy of Management, 17, 235-271.

Garst, H., Frese, M. \& Molenaar, P. C. M. (2000). The temporal factor of change in stressor-strain relationships: a growth curve model on a longitudinal study in East Germany. Journal of Applied Psychology, 85, 417-438.

Goldberg, D. \& Williams, P. (1991). A user's guide to the general health questionnaire. Windsor: NFER-Nelson.

Grebner, S., Semmer, N. K. \& Elfering, A. (2005). Working conditions and three types of well-beig: a longitudinal study with self-report and rating data. Journal of Occupational Health Psychology, 10, 31-43.

Griffiths, A. \& Schabracq, M. J. (2003). Work and health psychology as a scientific discipline: facing the limits of the nature science paradigm. In M. J. Schabracq, J. A. M. Winnubst \& C. L. Cooper (Eds.), Handbook of work and health psychology, (2 $2^{\text {nd }}$ ed.) (pp. 173-190). Chichester: John Wiley \& Sons.

Grunberg, L., Moore, S. \& Greenberg, E. S. (1998).Work stress and problem alcohol behavior: A test of the spillover model. Journal of Organizational Behavior, $19,487-502$.

Gunter, B. \& Furnham, A. (1996). Biographical and climate predictors of job satisfaction and pride in organizations. Journal of Psychology, 130, 192-208.

Hackman, J. R. \& Oldham, G. R. (1975). Development of the job diagnostic survey. Journal of Applied Psychology, 60, 159-170.

Hackman, J. R. \& Oldham, G. R. (1980). Work redesign. Reading, MA: Addison-Wesley.

Houkes, I., Janssen, P. P. M., De Jonge, J. \& Bakker, A. B. (2003). Specific determinants of intrinsic work motivation, emotional exhaustion and turnover intention: a multisample longitudinal study. Journal of Occupational and Organizational Psychology, 76, 427-450.

Ivanov, I. (2005). Mental health and working life. WHO Ministerial Conference on Mental Health Briefing Paper. Copenhagen: World Health Organization Regional Office for Europe.

James, L. R. \& Jones, A. P. (1974). Organizational climates: a review of theory and research. Psychological Bulletin, 81, 1096-1112.

James, L. R. \& Jones, A. P. (1976). Organizational structure: a review of structural dimensions and their conceptual relationships with individual attitudes and behavior. Organizational Behavior \& Human Decision Processes, 16, 74-113.

James, L. R. \& Tetrick, L. E. (1996). Confirmatory analytic tests of three causal models relating job perceptions to job satisfaction. Journal of Applied Psycho$\log y, 71,77-82$. 
James, L. R. \& Sells, S. B. (1981). Psychological climate: theoretical perspectives and empirical research. In D. Magnusson (Ed.), Toward a psychology of situations: an interactional perspective (pp. 275-295). Hillsdale, NJ: Erlbaum.

James, L. R., Hater, J. J., Gent, M. J. \& Bruni, J. R. (1979). Psychological climate: implications from cognitive learning theory and interactional psychology. Personnel Psychology, 30, 229-234.

James, L. R., James, L. A. \& Ashe, D. K. (1990). The meaning of organizations: the role of cognitions and values. In B. Schneider (Ed.), Organizational climate and culture (pp. 41-48). San Francisco: Jossey-Bass.

Jamison, C. S., Wallace, M. \& Jamison, P. L. (2004). Contemporary work characteristics, stress, and ill health. American Journal of Human Biology, 16, 43-56.

Johnson, J. W. \& LeBreton, J. M. (2004). History and use of relative importance indices in organizational research. Organizational Research Methods, 7, 238$-257$.

Jones, A. P. \& James, L. R. (1979). Psychological climate: dimensions and relationships of individual and aggregated work environment perceptions. Organizational Behavior \& Human Performance, 23, 201-250.

Jonge, J., Doormann, C., Janssen, P. P. M., Dollard, M., Landeweerd, J. A. \& Nijhuis, J. N. (2001). Testing reciprocal relationships between characteristics and psychological well-being: a cross-lagged structural equation model. Journal of Occupational and Organizational Psychology, 74, 29-46.

Jonge, J., Janssen, P. P. M. \& Breukelen, G. J. P. (1996). Testing the demand-control-support model among health care professionals: a structural equation model. Work and Stress, 10, 209-224.

Kaplan, G. A. \& Camacho, T. (1983). Perceived health and mortality: a nine-year follow-up of the human population laboratory cohort. American Journal Epidemiology, 117, 292-304.

Karasek, R. (1979). Job demands, jod decision latitude and mental strain: implications for job redesign. Administration Science Quarterly, 24, 285-307.

Kasl, S. V. (1986). Stress and disease in the workplace: a methodological commentary on the accumulated evidence. In M. F. Cataldo \& T. J. Coates (Eds.). Health and industry: a behavioral medicine perspective (pp. 52-85). New York: Wiley.

Kessler, R. C. (1987). The interplay of research design strategies and data analysis procedures in evaluating the effects of stress on health. In S. V. Kasl \& C. L. Cooper (Eds.), Research methods in stress and health psychology (pp. 113-140). Chichester, UK: Wiley.

Keyes, C. L. M. (2005). Mental illness and/or mental health? Investigating axioms of the complete state model of health. Journal of Consulting and Clinical Psychology, 73, 539-548.

Kopelman, R. E. (1985). Job redesign and productivity: a review of the evidence. National Productivity Review, 4, 237-255.

Kopelman, R. E., Brief, A. P. \& Guzzo, R. A. (1990). The role of climate and culture in productivity. In B. Scneider (Ed.), Organizational climate and culture (pp. 282-318). San Francisco: Jossey-Bass. 
LaRocco, J. M., House, J. S. \& French, J. R. P. (1980). Social support, occupational stress and health. Journal of Health and Social Behavior, 21, 202-218.

Larson, J. S. (1999). The conceptualization of health. Medical Care Research and Review, 56, 123-136.

Lawthorn, R., Patterson, M. \& West, M. (1996). Measuring organizational climate. Unpublished, Institute of Work Psychology, Sheffield.

Lazarus, R. S. (1999). Stress and emotion. London: Free Associations Books.

LeBreton, J. M., Ployhart, R. E. \& Ladd, R. T. (2004). A Monte Carlo comparison of relative importance methodologies. Organizational Research Methods, 7, 258-282.

Lee, R. T. \& Ashforth, B. E. (1996). A meta-analytic examination of the correlates of the three dimensions of job burnout. Journal of Applied Psychology, 81, 123-133.

Levi, L. (1982). Preventing work stress. Massachusetts: Adison-Wesley Reading.

Locke, E. A (1976). The nature and causes of job satisfaction. In M. D. Dunnette (Ed.), Handbook of industrial and organizational psychology. Chicago: Rand-McNally.

Lynch, J., Krause, N., Kaplan, G. A., Tuomilehto, J. \& Salonem, J. T. (1997). Workplace conditions, socioeconomic status, and the risk of mortality and acute myocardial infarction: the kuopio ischemic heart disease risk factor study. American Journal of Public Health, 87, 617-622.

Mathieu, J. E. \& Zajac, D. M. (1990). A review and meta-analysis of the antecedents, correlates, and consequences of organizational commitment. Psychological Bulletin, 108, 171-194.

McDaid, D., Curran, C. \& Knapp, M. (2005). Promoting mental well-being in the workplace: a european policy perspective. International Review of Psychiatry, $17,365-373$

McHorney, C., Ware, J. \& Raczeck, A. (1993). The MOS 36-item short-form health survey (SF-36). II: Psychometric and clinical tests of validity in measuring physical and mental health constructs. Med Care, 31, 247-263.

McHorney, C., Ware, J. \& Raczeck, A. (1993). The MOS 36-item short-form health survey (SF-36). II: Psychometric and clinical tests of validity in measuring physical and mental health constructs. Med Care, 31, 247-263.

Moscovici, S. (1984). The fenomenon of social representations. In R. M. Farr \& S. Moscovici (Eds.), Social representations (pp. 3-69). Cambridge: Cambridge University Press.

Mullarky, S., Wall, T. D., Warr, P. B., Clegg, C. W. \& Stride, C. B. (1999). Measures of job satisfaction, mental health and job-related well-being: $a$ bench-marking Manual. Sheffield: Institute of Work Psychology.

Nelson, D. L. \& Simmons, B. L. (2003). Health psychology and work stress: a more positive approach. In J. C. Quick \& L. E. Tetrick (Eds.), Handbook of occupational health psychology (pp. 97-119). Washington DC: American Psychological Association.

Ostroff, C., Kinicki, A. J. \& Tamkins, M. M. (2003). Organizational climate and culture. In W. C. Borman, D. R. Ilgen \& R. J. Klimoski (Eds.), Comprehensive 
handbook of psychology. Volume 12: Industrial and organizational psychology (pp. 365-402). Mahwah, NJ: Erlbaum.

Parker, C. P., Baltes, B. B., Young, S. A., Huff, J. W., Altman, R. A., Lacost, H. A. \& Roberts, J. E. (2003). Relationships between psychological climate perceptions and work outcomes: a meta-analytic review. Journal of Organizational Behavior, 24, 389-416.

Parker, S. K., Chmiel, N. \& Wall, T. D. (1997). Work characteristics and employee well-being with a context of strategic downsizing. Journal of Occupational Health Psychology, 2, 289-303.

Parker, S. K., Turner, N. \& Griffin, M. A. (2003). Designing healthy work. In D. A. Hofmann \& L. E. Tetrick (Eds.), Health and safety in organizations: a multilevel perspective (91-130). San Francisco, CA: Jossey-Bass.

Parker, S. K., Wall, T. D. \& Cordery, J. L. (2001). Future work design research and practice: towards an elaborated model of work design. Journal of Occupational and Organizational Psychology, 74, 413-440.

Parkes, K. R. (1995). The effects of objective workload on cognitive performance in a field setting: a two-period cross-over trial. Applied Cognitive Psychology, 9, S153-S157.

Patterson, M. G., Warr, P. B. \& West, M. A. (2004). Organizational climate and company performance: the role of employee affect and employee level. Journal of Occupational and Organizational Psychology, 77, 193-216.

Patterson, M. G., West, M. A., Shackleton, V. J., Dawson, J. F., Lawthom, R. L., Maitlis, S., Robinson, D. L. \& Wallace, A., M. (2005). Validating the organizational climate measure: links to managerial practices, productivity and innovation. Journal of Organizational Behavior, 26, 379-408.

Ramos, F. \& Sennfelt, (1996). Economia da saúde e saúde mental: os custos da depressão. Revista Portuguesa de Saúde Pública, 14, 53-68.

Reichers, A. E. \& Schneider, B. (1990). Climate and culture: an evolution of constructs. In B. Schneider (Ed.), Organizational climate and culture (pp. 5-39). San Francisco: Jossey-Bass.

Rentsch, J. (1990). Climate and culture: interaction and qualitative differences in organizational meanings. Journal of Applied Psychology, 75, 668-681.

Repetti, R. L. \& Cosmas, K. A. (1991). The quality of the social environment at work and job satisfaction. Journal of Applied Social Psychology, 21, 840-854.

Rizzo, J. R., House, R. J. \& Lirtzman, S. I. (1970). Role conflict and ambiguity in complex organizations. Administrative Science Quarterly, 15, 150-163.

Rousseau, D. M. (1988). The construction of climate in organizational research. In C. Cooper \& I. Robertson (Eds.). International review of industrial and organizational psychology (pp. 139-158). New York: Wiley.

Ryff, C. D. \& Singer, B. (1998). Human health: new directions for the new millennium. Psychological Inquiry, 9, 69-85.

Salovey, P., Rothman, A. J., Detweiler, J. B., \& Steward, W. T. (2000). The contours of positive human health. Psychological Inquiry, 9, 1-28. 
Schmidt, F. L. (1996). Statistical significance testing and cumulative knowledge in psychology: implications for training of researchers. Psychological Methods, 1, 115-129.

Schneider, B. \& Reichers, A. E. (1983). On the etiology of climates. Personnel Psychology, 36, 19-39.

Schneider, B. (2000). The psychological life in organizations. In N. M. Ashkanasy; C. P. Wilderom \& M. F. Peterson (Eds.), Handbook of organizational culture and climate (pp. xvii-xxi). Thousand Oaks, CA: Sage.

Seligman, M. E. P. \& Csikszentmihalyi, M. (2000). Positive psychology. American Psychologist, 55, 5-14.

Semmer, N. K. (2003). Individual differences, work stress and health. In M. J. Schabracq, J. A. M. Winnubst \& C. L. Cooper (Eds.), Handbook of work and health psychology, (2 ${ }^{\text {nd }}$ ed., pp. 83-120). Chichester: John Wiley \& Sons.

Semmer, N. K., Grebner, S. \& Elfering, A. (2004). Beyond self-report: using observational, physiological, and event-based measures in research on occupational stress. In P. L. Perrewé \& D. C. Ganster (Eds.), Research in occupational stress and well-being. Vol. 3. Emotional and physiological processes and positive intervention strategies (pp. 205-263). Amsterdam: Elsevier.

Shirom, A. (2003). The effects of work stress on health. In M. J. Schabracq, J. A. M. Winnubst \& C. L. Cooper (Eds.), Handbook of work and health psychology, ( $2^{\text {nd }}$ ed., pp. 63-82). Chichester: John Wiley \& Sons.

Siegrist, J. (1996). Adverse health effects of high-effort/low rward conditions. In C. L. Cooper (Ed.), Theories of organizational stress (pp. 190-204). Oxford: Oxford University Press.

Sonnentag, S. \& Frrese, M. (2004). Stress in organizations. In W. C. Borman, D. R. Ilgen \& J. R. kimoski (Eds.), Comprehensive handbook of psychology. Vol. 12: Industrial and organizational psychology. New York: John Wiley \& Sons.

Spector, P. E. (1997). Job satisfaction: application, assessment, causes, and consequences. Thousands Oaks, CA: Sage.

Spector, P. E. \& Brannick, M. T. (1995). The nature and effects of method variance in organizational research. In C. L. Cooper \& I. T. Robertson (Eds.), International review of industrial and organizational psychology (vol. 10, pp. 249-274). Chichester, Uk: Wiley.

Stansfeld, S., Head, J. \& Marmot, M. (2000). Work-related factors and ill-health: the withehall II study. HSE Contract Research report 266/2000. Sudbury: HSE Books.

Sutton, R. \& Kahn, R. L. (1986). Prediction, understanding and control as antidotes to organizational stress. In J. Lorsch (Ed.), Handbook of organizational behavior (pp. 392-412). Englewood Cliffs, NJ: Prentice-Hall.Sage Foundation.

Tetrick, L. E. \& LaRocco, J. M. (1987). Understanding, prediction, control as moderators of the relationship between perceived stress, satisfaction, and psychological well-being. Journal of Applied Psychology, 72, 538-543.

Theorelll, T. \& Karasek, R. (1996). Current issues relating to psychosocial job strain and cardiovascular disease research. Journal of Occupational Health Psycho$\log y, 1,9-26$. 
Van Veldhoven, M., Taris, T. W. \& De Jonge, J. (2005). The relationship between work characteristics and employee health and well-being: how much complexity do we really need? International Journal of Stress Managemen, 12, 3-28.

Virtanen, P., Liukkonen, V., Virtanen, M., Vahtera, J. \& Ferrieg, J. (2005). Labor markets trajectories and health: a four-year follow-up study of Initially fixed-term employees. American Journal of Epidemioloy, 16, 840-846.

Viswervaran, C., Sanchex, J. I. \& Fisher, J. (1999). The role of social support in the process of work stress: a meta-analysis. Journal of Vocational Behaviour, 54, 314-334.

Wall, T. D., Kemp, N. J., Jackson, R. P. \& Clegg, C. W. (1986). Outcomes of autonomous work groups: a long term field experiment. Academy of Management Journal, 29, 280-304.

Wanous, J. P., Reichers, E. \& Hudy, M. J. (1997). Overall job satisfaction: how good are single-item measures? Journal of Applied Psychology, 82, 247-252.

Warr, P. (1990). Decision latitude, job demands and employee well being. Work and Stress, 4, 285-294.

Warr, P. (1990a). The measurement of well-being and other aspects of mental-health. Journal of Occupational Psychology, 52, 129-148.

Warr, P. (1999). Well-being and the workplace. In D. Kahneman, E. Diener \& N. Schwartz (Eds.), Well-being: the foundations of hedonic psychology (pp. 392-412). New York: Russell Sage Foundation.

Warr, P., Cook, J. \& Wall, T. (1979). Scales for the measurement of some work attitudes and aspects of psychological well-being. Journal of Occupational Psychology, 52, 129-148.

Weick, K. E. (1995). Sensemaking in organizations. Thousands Oaks, CA: Sage.

Wiener, Y. (1982). Commitment in organizations: a normative view. Academy of Management Review, 7, 418-428).

Williams, L. J. \& Brown, B. K. (1994). Method variance in organizational behavior and human resources research: effects on correlations, path coefficients, and hypothesis testing. Organizational Behavior and Human Decision Processes, 57, 185-209.

Winefield, A. H., Tiggemann, M. \& Goldney, R. D. (1988). Psychological concomitants of satisfactory employment and unemployment in young people. Social Psychiatry \& Psychiatric Epidemiology, 223, 149-157.

World Health Organization (WHO) (1948). Preamble to the Constitution of the World Health Organization as adopted by the International Health Conference, New York, 19-22 June 1946; signed on 22 July 1946 by the representatives of sixty-one states (Official Records of the World Health Organization, n. ${ }^{\circ} 2$, p. 100) and entered into force on 7 April 1948.

World Health Organization (WHO) (1996). World health report: Fighting diseases fostering development. Geneva: World Health Organization.

World Health Organization (WHO) (2001). World health report 2001. Geneva: World Health Organization. 
Wright, T. A. \& Bonett, D. G. (1992). The effect of turnover on work satisfaction and mental health: support for a situational perspective. Journal of Organizational Behavior, 13, 603-615.

Zapf, D., Dormann, C. \& Frese, M. (1996). Longitudinal studies in organizational stress research: a review of the literature with reference to methodological issues. Journal of Occupational Health Psychology, 1, 145-169. 